\title{
Independent Variables for Determining the \\ Cumulative Live Birth Rates of Aged Patients With Polycystic Ovary Syndrome or Tubal Factor Infertility: A Retrospective Cohort Study
}

\section{Pingping Kong}

Zhengzhou University Third Hospital and Henan Province Women and Children's Hospital

\section{Zhiying Xiao}

Zhengzhou University Third Hospital and Henan Province Women and Children's Hospital Junyan Zhang

Bothwin clinical study consultant

Jingfang He

Bothwin clinical study consultant

\section{Wenjun Geng}

Zhengzhou University Third Hospital and Henan Province Women and Children's Hospital Junfang Yan

Zhengzhou University Third Hospital and Henan Province Women and Children's Hospital

\section{Simin Sun}

Zhengzhou University Third Hospital and Henan Province Women and Children's Hospital

\section{Mingkun Mu}

Zhengzhou University Third Hospital and Henan Province Women and Children's Hospital

\section{Xiaofang Du}

Zhengzhou University Third Hospital and Henan Province Women and Children's Hospital

\section{Xingling Wang}

Zhengzhou University Third Hospital and Henan Province Women and Children's Hospital

Yichun Guan ( $\nabla$ lisamayguan1@126.com )

Zhengzhou University Third Hospital and Henan Province Women and Children's Hospital https://orcid.org/0000-0002-0312-3984

\section{Research}

Keywords: polycystic ovary syndrome, advanced age, $\geq 35$ years, cumulative live birth rates, tubal factor infertility, in vitro fertilization, intracytoplasmic sperm injection 
DOI: https://doi.org/10.21203/rs.3.rs-143361/v1

License: (c) (1) This work is licensed under a Creative Commons Attribution 4.0 International License. Read Full License 


\section{Independent Variables for Determining the Cumulative Live Birth Rates of Aged Patients with Polycystic Ovary Syndrome or Tubal Factor Infertility: A Retrospective Cohort Study}

1 Pingping Kong ${ }^{1}$, Zhiying Xiao', Junyan Zhang², Jingfang He², Wenjun Geng', Junfang Yan',

2 Simin Sun ${ }^{1}$, Mingkun Mu${ }^{1}$, Xiaofang Du ${ }^{1}$, Xingling Wang ${ }^{1}$, Yichun Guan ${ }^{1 *}$,

$3{ }^{1}$ The Reproduction Center, the Third Affiliated Hospital of Zhengzhou University, Zhengzhou, China

$4 \quad{ }^{2}$ Bothwin Clinical Study Consultant, WA, USA

5 * Correspondence:

6 Yichun Guan

7 lisamayguan1@126.com

\section{Abstract}

9 Background: Women with polycystic ovary syndrome (PCOS) have a higher ovarian reserve and number of oocytes retrieved than women with tubal infertility. To assess whether women of advanced age ( $\geq 35$ years) with PCOS have the same cumulative live birth rate (CLBR) as their agematched controls with tubal factor infertility and to determine the influencing factors on the CLBRs

13 of aged women.

14 Methods: A total of 190 women of advanced age ( $\geq 35$ years) with PCOS and 627 women with tubal factor infertility were included in our study. All patients underwent their first fresh cycles and subsequent frozen cycles in our centre from 2007 to 2018. To determine independent influencing factors on the CLBRs of these aged patients, a multivariable Cox regression model of CLBR according to the transfer cycle type was constructed.

Results: The Cox regression model of the CLBRs indicated that there was no significant difference between the PCOS group and the tubal infertility group in terms of advanced age (HR, 0.96; 95\% CI, 0.77 1.20). The CLBR significantly decreased for women of advanced reproductive age up to 37 years of age (HR, 0.65; 95\% CI, 0.53 0.80). The CLBR increased by $31 \%$ when more than ten oocytes were retrieved (HR, 1.31; 95\% CI, 1.08 1.59). In addition to age and the number of oocytes, the addition of recombinant LH was an independent factor that increased the CLBRs of the women of advanced age (HR, 1.25; 95\% CI, 1.03 1.53).

Conclusions: Despite the higher number of oocytes retrieved in PCOS patients, the reproductive window is not extended for PCOS patients compared with tubal factor infertility patients. Age, the number of oocytes retrieved and supplementation with LH play crucial roles in the CLBRs of patients of advanced age ( $\geq 35$ years).

30 Keywords: polycystic ovary syndrome, advanced age, $\geq 35$ years, cumulative live birth rates, 31 tubal factor infertility, in vitro fertilization, intracytoplasmic sperm injection

\section{Introduction}


Polycystic ovary syndrome (PCOS) is a widespread reproductive disorder that encompasses many associated health conditions and impacts various metabolic processes. This condition leads to an increased risk of insulin resistance (IR), type 2 diabetes, obesity, and cardiovascular disease. The aetiology of PCOS remains unclear ${ }^{(1)}$. The three main phenotypic characteristics of this condition are hyperandrogenism, polycystic ovaries, and ovulatory dysfunction ${ }^{(2)}$.

PCOS is the most common cause of menstrual irregularity that leads to infertility. Among all cases comprising couples seeking treatment for infertility, $30 \%$ are due to anovulation ${ }^{(3)}$. It is estimated that $90 \%$ of anovulation cases are due to PCOS ${ }^{(4)}$. The most common treatments used for ovulation induction are clomiphene citrate (CC) and letrozole ${ }^{(5)}$. For PCOS patients who exhibit CC resistance and letrozole failure, assisted reproductive technology (ART) may play a role in helping them to achieve pregnancy ${ }^{(4,6)}$. Moreover, in vitro fertilization (IVF) and intracytoplasmic sperm injection (ICSI) are the most popular ART treatments.

Traditionally, the success rates of IVF/ICSI have been reported in terms of the number of live births per embryo transfer cycle. With the increasing number of frozen-thawed cycles, it has become essential that we report the outcomes not only of fresh embryo transfers but also of frozen embryo transfers as a complete measure of the success of IVF/ICSI treatment ${ }^{(7)}$. The cumulative live birth rate (CLBR), which includes fresh and subsequent frozen-thawed embryo transfer (FET) cycles, may be one of the most meaningful outcome from the patient's perspective ${ }^{(8)}$. Women with PCOS have a higher ovarian reserve and number of oocytes retrieved than women with tubal infertility ${ }^{(9)}$. Previous research has shown that the higher the number of oocytes retrieved is, the higher the CLBR will be ${ }^{(8)}$. It seems that women with PCOS should have a higher CLBR. However, with fresh cycles, women with PCOS over the age of 40 have similar clinical pregnancy and live birth rates to those of women with tubal factor infertility ${ }^{(9)}$.

Several studies utilized CLBR of PCOS patients as their primary outcome measure. Nevertheless, some of these studies have taken into account different PCOS phenotypes instead of advanced age, such as hyperandrogenic PCOS phenotypes ${ }^{(10)}$ and female obesity ${ }^{(11)}$. While others focused on the CLBRs of PCOS women who underwent IVM ${ }^{(12)}$. Samer Tannus et al. studied CLBR in PCOS women over the age of 40 with only a small number of PCOS women included ${ }^{(13)}$. Moreover, few studies have focused on the CLBRs of women of advanced age ( $\geq 35$ years) with PCOS who are treated by IVF/ICSI. Therefore, we aimed to determine whether aged women with PCOS have the same CLBRs as women with tubal infertility and to characterize the influencing factors on the

64 CLBRs of aged women with infertility.

\section{$2 \quad$ Materials and methods}

\subsection{Patients}

This retrospective cohort study included 817 patients who had undergone IVF/ICSI in our centre between July 2007 and September 2018. Written approval for this study was obtained from the Ethics

All patients underwent their first fresh cycles and subsequent frozen cycles in our centre and the patient's age was $\geq 35$ years. According to the cause of infertility, patients were divided into two groups: the PCOS group (190 PCOS patients) and tubal infertility group (627 patients). PCOS was diagnosed according to criteria from the Rotterdam European Society for Human Reproduction and Embryology/American Society for Reproductive Medicine-Sponsored PCOS Consensus Workshop Group ${ }^{(14)}$. The patients in the tubal infertility group were with or without male factor infertility. The 
exclusion criteria were as follows: 1) Infertile patients with endometriosis, uterine submucosal fibroids and uterine malformations; 2) infertile patients with endocrine or metabolic diseases; 3) cycles with oocyte donation; or 4) cycles with pre-implantation genetic testing for aneuploidy (PGTA). Patients who did not have a live birth and had remaining embryos were also excluded. The patients' characteristics, including age, body mass index (BMI), duration of infertility, reproductive endocrine hormone levels, fresh cycle outcomes and CLBRs, were evaluated and recorded. An overview of the patient selection and grouping is provided in Figure 1. The endpoint of this study was the first live birth of the patient or no remaining embryos for the patient. Any missing data were excluded from the analysis.

\subsection{Methods}

\subsubsection{Treatment protocol}

The protocol used for controlled ovarian stimulation (COS) was a long gonadotrophin-releasing hormone $(\mathrm{GnRH})$-agonist protocol for IVF or ICSI. All patients were treated with recombinant and/or urinary gonadotrophins (Gonal-F, Merck Serono, Germany; or Puregon, Organon, Netherlands; or hMG, Livzon, China) with or without supplementation with recombinant luteinizing hormone (rLH) (Luveris, Merck Serono, Germany). The initial dose ranged from 112.5 to 300 IU per day depending on age, BMI and the results of ovarian reserve tests. During stimulation, the ovarian response was monitored by serial transvaginal ultrasound and serum hormone level measurements.

Ovulation was triggered with recombinant HCG (r-HCG, Ovidrel, Merck Serono, Germany) in all cases, when at least three follicles $\geq 18 \mathrm{~mm}$ in diameter were observed. Oocytes were retrieved 36-38 $\mathrm{h}$ later. Embryo transfer was carried out under ultrasound guidance three or five days after oocyte retrieval. Supernumerary embryos were cryopreserved following vitrification standard protocols.

For luteal phase support, the use of vaginal progesterone gel (Crinone, Merck Serono, Germany: 90 mg every $24 \mathrm{~h}$ ) or micronized natural progesterone capsules (Utrogestan, Besins, Belgium: $200 \mathrm{mg}$ every $8 \mathrm{~h}$ ) combined with oral dydrogesterone (Duphaston, Abbott, Netherlands: $10 \mathrm{mg}$ every $12 \mathrm{~h}$ ) was started on the day of retrieval and continued until the beta-hCG measurement showed that the patient was negative for beta-hCG or until the tenth week of pregnancy.

\subsubsection{Endometrial preparation protocol}

For FET cycles, endometrial preparation for frozen-thawed cycles involved hormonal replacement therapy or ovulation induction cycles. In the hormone replacement cycle, oral oestrogens (Progynova, Bayer, Germany) and micronized progesterone were given with or without pituitary suppression with triptorelin depot $3.75 \mathrm{mg}$ (Diphereline, Epsen, France). In the ovulation induction cycles, patients were administered 2.5 to $5 \mathrm{mg}$ of letrozole daily for five days. If the diameter of the dominant follicle was $\geq 18-20 \mathrm{~mm}$, ovulation was then stimulated with $10,000 \mathrm{IU}$ of hCG (hCG, Livzon, China) ${ }^{(15)}$.

\subsection{Outcome measures}

The primary outcome was CLBRs, defined as the delivery of a live born infant (after 28 weeks of gestation) in the fresh or the subsequent frozen-thawed cycles. Only the first delivery was considered in the analysis.

\subsection{Statistical analysis}


115 Patients were divided into two groups according to the cause of infertility (PCOS vs tubal infertility).

116 For continuous variables, Student's t-test was used for data with homogeneous variance, and the

117 Mann-Whitney test was used for data with heterogeneous variance. The $\chi^{2}$ test was used for

118 categorical variables. Variables with greater clinical importance or with large variances were selected

119 for further assessment.

120 The cohort was analyzed using multivariable Cox regression analyses to determine independent risk factors of CLBR. Time was transfer cycles at risk of CLBR. Transfer cycle was determined from the time a woman started trying to transfer, not from the time of controlled ovarian stimulation. The end point of time was the transfer cycle to obtain a live birth or the last available embryo transfer cycle. Risk factors were infertility cause, infertility duration, age, the number of oocytes retrieved, rLH supplementation, and BMI, among others. We adopted the receiver operating characteristic (ROC) curve to find the best point of sensitivity and specificity as the cut off value. In order to application to clinical practice, the nearest integers were adopted. To examine infertility cause, patients were categorized into the PCOS and tubal groups. To examine age, patients were categorized into two age groups (35-37 and >37 years). To examine the number of retrieved oocytes, patients were categorized into two groups $(\leq 10$ and $>10)$. The CLBRs after IVF and for certain study factors are presented as the hazard ratio (HR) with the $95 \%$ confidence interval (CI). Analyses were performed using Stata

13213.1 (Serial number: 401306302851).

\section{$133 \quad 3 \quad$ Results}

\section{$134 \quad 3.1 \quad$ Tubal factor infertility and PCOS groups}

135 As shown in Table 1, although the basal testosterone (T) levels were stable across the two groups, a 136 higher BMI $(24.43 \pm 3.40$ vs $23.49 \pm 3.11, P<0.001)$, higher $\mathrm{LH} / \mathrm{FSH}$ ratio $(1.01 \pm 1.10$ vs $0.59 \pm 0.27$, $137 P<0.001)$ and longer duration of infertility $(5.78 \pm 4.11$ vs $5.15 \pm 4.33, P=0.007)$ were observed in the PCOS group than in the tubal factor group. In addition, younger age (36.93 \pm 2.19 vs $38.02 \pm 2.83$, $P<0.001)$, a lower total gonadotropin (Gn) dose $(2712.17 \pm 1247.43$ vs $3320.32 \pm 1040.29, P<0.001)$, a higher number of oocytes retrieved $(12.87 \pm 6.67$ vs $10.05 \pm 5.34, P<0.001)$ and a higher rate of rLH supplementation $(57.89 \%$ vs $36.84 \%, P<0.001)$ were observed in the PCOS group. However, no significant difference was detected in the high-quality embryo rate $(0.41 \pm 0.28$ vs $0.38 \pm 0.31$, $P=0.297$ ) between the two groups.

\section{$144 \quad 3.2 \quad$ Live-birth group and the non-live-birth group}

145 Regarding all transfer cycles (fresh and frozen) outcomes, all patients were divided into two groups 146 depending on whether a live birth was achieved(Table 2). Although the BMI and duration of 147 infertility did not differ significantly between the two groups, the basal T levels (19.60 \pm 13.18 vs $14817.86 \pm 10.58, P=0.042)$ and $\mathrm{LH} / \mathrm{FSH}$ ratio $(0.73 \pm 0.74$ vs $0.63 \pm 0.48, P=0.038)$ were higher in the 149 live-birth group. Nevertheless, younger age $(37.10 \pm 2.20$ vs $38.64 \pm 3.11, P<0.001)$ and lower total Gn dose $(3006.47 \pm 1123.15$ vs $3406.67 \pm 1078.25, P<0.001)$ were observed in the live-birth group. Moreover, a higher proportion of PCOS patients $(28.17 \%$ vs $16.76 \%, P<0.001)$, rate of rLH supplementation $(46.45 \%$ vs $35.51 \%, P=0.002)$, number of oocytes retrieved $(12.22 \pm 5.96$ vs $8.71 \pm 4.91, P<0.001)$ and rate of high-quality embryos $(0.48 \pm 0.32$ vs $0.39 \pm 0.36, P<0.001)$ were evident in the live-birth group than in the non-live-birth group.

\section{$155 \quad 3.3$ Cumulative live birth rates}


As shown in Table 3, 465 live births were observed. The multivariable Cox regression analyses of all transfer cycles (fresh and frozen) concerning timescale showed that the CLBRs of the patients of advanced age with PCOS were not statistically significantly higher than those of the patients with tubal factor infertility (HR, 0.96; 95\% CI, 0.77 1.20, $P=0.727$ ). Compared with that of patients under 37 years old, the CLBR of patients older than 37 years decreased by $35 \%$, which was statistically significant (HR, 0.65; 95\% CI, $0.53 \sim 0.80, P<0.001$ ). The CLBR increased by $31 \%$ when more than ten oocytes were retrieved (HR, 1.31; 95\% CI, 1.08 1.59, $P=0.005)$. Recombinant $\mathrm{LH}$ supplementation during ovarian stimulation increased CLBR by $25 \%$ (HR 1.25; 95\% CI, $1.03 \sim 1.53$, $P=0.022)$.

\section{Discussion}

According to our multivariable Cox regression analyses, age, number of oocytes retrieved and supplementation with LH play crucial roles in the CLBRs of patients of advanced age ( $\geq 35$ years). Despite the higher number of oocytes retrieved in PCOS patients, the reproductive window is not extended in patients of advanced age with PCOS compared to those with tubal factor infertility.

170 Over recent decades, IVF/ICSI protocols have continued to evolve to improve outcomes. However, there is still a linear decline in the success rate of ART with patient age. In general, advanced age is defined as $\geq 35$ years, and oocyte depletion of the female follicular pool continues from the embryonic stage. After age 35, and especially after age 37, follicle numbers decline bi-exponentially rather than as a simple exponential function of age ${ }^{(16)}$. A previous study also found that the CLBR was age dependent and declined from over $50 \%$ for women who were $\leq 37$ years old to $34.1 \%$ for women who were $38-40$ years old to $17.7 \%$ for women who were $41-42$ years old ${ }^{(17)}$. Our study is in complete agreement with the study above, demonstrating that compared with that of women younger than 37 years, the CLBR of women over 37 years old significantly decreased by $35 \%$.

Additionally, ageing may also affect the CLBR by affecting oocyte quality. Mitochondria play a central role in follicular atresia and can be the main target of ooplasmic factors that determine oocyte quality. Age-related mitochondrial DNA (mtDNA) instability, which leads to the accumulation of mtDNA mutations in the oocyte, may play a key role in the deterioration of oocyte quality and the risk of transmitting mitochondrial abnormalities to the offspring ${ }^{(18)}$. Previous studies have shown that the incidence of oocyte aneuploidy increases with age ${ }^{(19,20)}$. Embryo quality may be the main factor affecting the live birth rate after elective single-embryo transfer in fresh stimulation cycles ${ }^{(21)}$.

In addition to age, the number of retrieved oocytes is an important factor in the CLBR. An increased number of oocytes retrieved improves the pregnancy rates of women undergoing IVF/ICSI, not only by increasing the number of available embryos but also by allowing extended embryo culture and enabling the selection of the best-quality embryo for transfer ${ }^{(22)}$. Furthermore, after the fresh embryo transfer, there are more opportunities for FET, which may increase the CLBR. Our study demonstrated that the CLBR increased by $31 \%$ when more than ten oocytes were retrieved (HR, $1.31 ; 95 \% \mathrm{CI}, 1.08 \sim 1.59)$. This is in agreement with a multivariate logistic regression analysis showing that the number of oocytes retrieved was an independent predictive factor $(P<0.001)$ for the CLBR after adjustments for fertilization rate, age, day of fresh embryo transfer and insemination $\operatorname{method}^{(23)}$.

Many factors may directly or indirectly impair the competence of maturing oocytes, resulting in a lower pregnancy rate for patients with PCOS. The identified extra-ovarian factors include hyperandrogenaemia and hyperinsulinaemia, while intra-ovarian factors comprise members of the 
199

200

201

202

203

204

205

206

207

208

209

210

211

212

213

214

215

216

217

218

219

220

221

222

223

224

225

226

227

228

229

230

231

232

233

234

235

236

237

238

239

240

241

epidermal, fibroblast, insulin-like and neurotrophin families of growth factors, as well as cytokines. Any abnormality may negatively affect the granulosa cell-oocyte interaction, oocyte maturation and potential embryonic developmental competence, which contribute to unsuccessful outcomes for PCOS patients undergoing assisted reproduction ${ }^{(24)}$. In PCOS patients, the mitochondrial membrane potential, ATP content, mtDNA copy number, and HIF-1 $\alpha$ mRNA and protein levels in granulosa cells decreased, while the levels of reactive oxygen species increased, affecting the switch of energy metabolic to glycolytic. That might be correlated to the low oocyte competence of $\operatorname{PCOS}^{(25)}$. Women with PCOS were at an increased risk of miscarrying a chromosomally aberrant embryo/fetus compared with non-PCOS controls during ART ${ }^{(26)}$. While Li et al. ${ }^{(27)}$ found that among aged women $(\geq 35$ years), the PCOS patients exhibited a higher CLBR than the non-PCOS patients, which is not consistent with our outcome. Whether PCOS is an influencing factor in aged patients, further clinical studies on larger sample sizes and mechanism studies are needed.

We also investigated the influence of rLH supplementation on the CLBRs of women of advanced age. With ageing, the number of LH receptors and LH receptor sensitivity decrease due to paracrine deficiency ${ }^{(28)}$. In ovulation cycles, LH is essential for steroid production and the development of follicles ${ }^{(29)}$. It has also been proposed that the endogenous LH level increases with age and that the LH concentration remains high after pituitary downregulation ${ }^{(30)}$. Whether it is necessary to supply rLH during COS remains unclear.

Schwarze et al. ${ }^{(31)}$ found that there was no association between supplementation with LH and an increased mean number of inseminated metaphase-II oocytes, an increase in the delivery rate, or changes in the miscarriage rate, and the authors suggested that LH supplementation had little impact on the outcome of ART. However, their cohort study primarily focused on a general population of infertile women instead of women of advanced age. Hill et al. ${ }^{(32)}$ reviewed seven trials that focused only on patients of advanced reproductive age and reported that the supplementation with rLH in ART cycles may improve the implantation and clinical pregnancy rates of these patients. This is consistent with our study, which showed that rLH supplementation increased the CLBRs by $25 \%$.

To the best of our knowledge, this is one of the first few studies to determine the CLBRs of women of advanced age with PCOS. A major strength of the current study is its attempt to explore the CLBRs of women of advanced age with PCOS. PCOS is one of the most prevalent endocrine disorders. The reproductive lifespan of women with PCOS may be, on average, two years longer than that of normo-ovulatory women ${ }^{(33)}$. Compared with non-PCOS patients, PCOS patients show a slow decline in ovarian reserve function and delayed physiological ageing of their ovarian follicles ${ }^{(34)}$. Therefore, it seems that the PCOS group would have a higher CLBR.

However, the available data show the opposite. Despite the higher oocyte yield in all age groups of women with PCOS, patients over age 40 with PCOS who underwent fresh cycles had similar clinical pregnancy and live birth rates to those of the women with tubal factor infertility who also underwent fresh cycles ${ }^{(9)}$. These findings suggest that the reproductive window is not extended by PCOS as patients age and that infertile patients should be treated promptly, despite indicators of high ovarian reserve ${ }^{(9)}$. Fertility in patients with PCOS is maintained until the age of 38 years with the use of IVF. Afterward, the pregnancy rate decreases, although the number of oocytes retrieved by IVF remains stable ${ }^{(35)}$. We demonstrated that the CLBR of PCOS patients was comparable to that of the tubal factor infertility patients of advanced age, which may not be consistent with li et al. ${ }^{(27)}$, but we adjusted for more critical confounders in our analysis. 
This study expands on previous research, but it has several limitations, most of which are associated with the retrospective study design. First, the compared groups may have differed in terms of several baseline characteristics. Nevertheless, the effects of these differences would be minimal given that these factors were analysed in multivariable Cox regression analyses. Second, due to the incompleteness of the original data, more detailed characterization of presenting features of the PCOS patients that could potentially affect the pregnancy outcome were not included in the analysis. Finally, the live birth rate is closely related to the rate of aneuploid embryos assessed by PGT-A and mitochondrial DNA copy number tested with next generation sequencing (NGS). We did not review the data of aneuploid embryos and mitoscoreo at admission. Whether or not women of advanced age with PCOS have more aneuploid embryos than those with tubal infertility should be assessed.

\section{2}

253

254

255

256

257

258

259

260

261

262

263

264

265

266

267

268

269

270

271

\section{2}

273

274

275

276

277

\section{Conclusion}

Even though their ovarian reserve is high, women of advanced age with PCOS should be given more attention with the help of ART. Despite the higher number of oocytes retrieved, the reproductive window is not extended for patients with PCOS compared with those with tubal factor infertility. Age, the number of oocytes retrieved and supplementation with LH play crucial roles in the CLBRs of patients of advanced age ( $\geq 35$ years).

\section{Acknowledgments}

The abstract had been accepted for oral presentation at the IFFS 2019 Congress in Shanghai, China (36). The authors thank the participants and staff of the Reproduction Center for their valuable contributions to this research. The authors would like to acknowledge the professional manuscript services of American Journal Experts.

\section{Author contributions}

YG developed the conceptual framework and research protocol for the study. PK, ZX and WG conducted the data collection. JZ and JH carried out the statistical analysis. JY, SS, MM, and XW interpreted the data. XD edited the flowchart and tables. PK and ZX drafted the manuscript. YG made major revisions. All authors approved the final version of the manuscript.

\section{Funding}

No funding was required or obtained.

\section{Availability of data and materials}

Not applicable.

\section{Ethics approval and consent to participate}

Institutional Review Board approval was obtained from the Ethics Committee of The Third Affiliated Hospital of Zhengzhou University.

\section{Consent for publication}

Not applicable.

\section{Competing interests}


278 Author Junyan Zhang and Jingfang He were employed by the company Bothwin Clinical Study

279 Consultant. The remaining authors declare that the research was conducted in the absence of any 280 commercial or financial relationships that could be construed as a potential conflict of interest. 


\section{References}

283 1. Barthelmess EK, Naz RK. Polycystic ovary syndrome: current status and future perspective.

284 Front Biosci (Elite Ed). 2014;6:104-19.

285 2. Rotterdam ESHRE ASRM-Sponsored PCOS Consensus Workshop Group. Revised 2003

286 consensus on diagnostic criteria and long-term health risks related to polycystic ovary syndrome.

287 Fertil Steril. 2004;81(1):19-25.

288 3. Balen A, Michelmore K. What is polycystic ovary syndrome? Are national views important?

289 Hum Reprod. 2002;17(9):2219-27.

290 4. Balen AH, Rutherford AJ. Managing anovulatory infertility and polycystic ovary syndrome.

291 BMJ. 2007;335(7621):663-6.

292 5. Casper RF. Letrozole versus clomiphene citrate: which is better for ovulation induction?

293 Fertil Steril. 2009;92(3):858-9.

294 6. Balen AH, Morley LC, Misso M, Franks S, Legro RS, Wijeyaratne CN, et al. The

295 management of anovulatory infertility in women with polycystic ovary syndrome: an analysis of the

296 evidence to support the development of global WHO guidance. Hum Reprod Update.

297 2016;22(6):687-708.

298 7. Maheshwari A, McLernon D, Bhattacharya S. Cumulative live birth rate: time for a

299 consensus? Hum Reprod. 2015;30(12):2703-7.

300 8. Devesa M, Tur R, Rodriguez I, Coroleu B, Martinez F, Polyzos NP. Cumulative live birth

301 rates and number of oocytes retrieved in women of advanced age. A single centre analysis including

3024500 women $>$ /=38 years old. Hum Reprod. 2018;33(11):2010-7.

303 9. Kalra SK, Ratcliffe SJ, Dokras A. Is the fertile window extended in women with polycystic

304 ovary syndrome? Utilizing the society for assisted reproductive technology registry to assess the

305 impact of reproductive aging on live-birth rate. Fertil Steril. 2013;100(1):208-13.

306 10. De Vos M, Pareyn S, Drakopoulos P, Raimundo JM, Anckaert E, Santos-Ribeiro S, et al.

307 Cumulative live birth rates after IVF in patients with polycystic ovaries: phenotype matters. Reprod

308 Biomed Online. 2018;37(2):163-71.

309 11. Ding W, Zhang FL, Liu XC, Hu LL, Dai SJ, Li G, et al. Impact of Female Obesity on

310 Cumulative Live Birth Rates in the First Complete Ovarian Stimulation Cycle. Front Endocrinol

311 (Lausanne). 2019;10:516.

312 12. Ho VNA, Pham TD, Le AH, Ho TM, Vuong LN. Live birth rate after human chorionic

313 gonadotropin priming in vitro maturation in women with polycystic ovary syndrome. J Ovarian Res.

$3142018 ; 11(1): 70$.

315 13. Tannus S, Tan J, Son WY, Dahan MH. Prevalence, clinical characteristics, and reproductive 316 outcomes of polycystic ovary syndrome in older women referred for tertiary fertility care. Arch

317 Gynecol Obstet. 2018;297(4):1037-42.

318 14. Rotterdam ESHRE ASRM-Sponsored PCOS Consensus Workshop Group. Revised 2003

319 consensus on diagnostic criteria and long-term health risks related to polycystic ovary syndrome

320 (PCOS). Hum Reprod. 2004;19(1):41-7.

321 15. Guan Y, Fan H, Styer AK, Xiao Z, Li Z, Zhang J, et al. A modified natural cycle results in

322 higher live birth rate in vitrified-thawed embryo transfer for women with regular menstruation. Syst

323 Biol Reprod Med. 2016;62(5):335-42. 
16. Faddy MJ, Gosden RG, Gougeon A, Richardson SJ, Nelson JF. Accelerated disappearance of ovarian follicles in mid-life: implications for forecasting menopause. Hum Reprod. 1992;7(10):13426.

327 17. De Neubourg D, Bogaerts K, Blockeel C, Coetsier T, Delvigne A, Devreker F, et al. How do 328 cumulative live birth rates and cumulative multiple live birth rates over complete courses of assisted reproductive technology treatment per woman compare among registries? Hum Reprod. 2016;31(1):93-9.

331 18. May-Panloup P, Boucret L, de la Barca CJM, Desquiret-Dumas V, Ferre-L'Hotellier V, 332 Moriniere C, et al. Ovarian ageing: the role of mitochondria in oocytes and follicles. Hum Reprod 333 Update. 2016;22(6):725-43.

334 19. Pellestor F, Andreo B, Arnal F, Humeau C, Demaille J. Maternal aging and chromosomal abnormalities: new data drawn from in vitro unfertilized human oocytes. Hum Genet. 2003;112(2):195-203.

337 20. Franasiak JM, Forman EJ, Hong KH, Werner MD, Upham KM, Treff NR, et al. The nature of 338 aneuploidy with increasing age of the female partner: a review of 15,169 consecutive trophectoderm biopsies evaluated with comprehensive chromosomal screening. Fertil Steril. 2014;101(3):656-63 e1.

340 21. Niinimaki M, Veleva Z, Martikainen H. Embryo quality is the main factor affecting cumulative live birth rate after elective single embryo transfer in fresh stimulation cycles. Eur $\mathbf{J}$ Obstet Gynecol Reprod Biol. 2015;194:131-5.

22. Fauser BC, Devroey P, Macklon NS. Multiple birth resulting from ovarian stimulation for subfertility treatment. Lancet. 2005;365(9473):1807-16.

23. Drakopoulos P, Blockeel C, Stoop D, Camus M, de Vos M, Tournaye H, et al. Conventional ovarian stimulation and single embryo transfer for IVF/ICSI. How many oocytes do we need to maximize cumulative live birth rates after utilization of all fresh and frozen embryos? Hum Reprod. 2016;31(2):370-6.

24. Qiao J, Feng HL. Extra- and intra-ovarian factors in polycystic ovary syndrome: impact on oocyte maturation and embryo developmental competence. Hum Reprod Update. 2011;17(1):17-33. 1alpha signaling in granulosa cells of polycystic ovary syndrome. Endokrynol Pol. 2020.

26. Li Y, Wang L, Xu J, Niu W, Shi H, Hu L, et al. Higher chromosomal aberration rate in miscarried conceptus from polycystic ovary syndrome women undergoing assisted reproductive treatment. Fertil Steril. 2019;111(5):936-43 e2. Polycystic Ovary Syndrome. Front Endocrinol (Lausanne). 2019;10:834. randomized, clinical trial. Hum Reprod. 2009;24(2):349-59.

362 29. Chung K, Krey L, Katz J, Noyes N. Evaluating the role of exogenous luteinizing hormone in 363 poor responders undergoing in vitro fertilization with gonadotropin-releasing hormone antagonists.

364 Fertil Steril. 2005;84(2):313-8. 
365 30. Weghofer A, Munne S, Brannath W, Chen S, Barad D, Cohen J, et al. The impact of LH-

366 containing gonadotropin stimulation on euploidy rates in preimplantation embryos: antagonist cycles.

367 Fertil Steril. 2009;92(3):937-42.

368 31. Schwarze J-E, Crosby JA, Zegers-Hochschild F. Addition of neither recombinant nor urinary

369 luteinizing hormone was associated with an improvement in the outcome of autologous in vitro

370 fertilization/intracytoplasmatic sperm injection cycles under regular clinical settings: a multicenter

371 observational analysis. Fertil Steril. 2016;106(7):1714-7.e1.

372 32. Hill MJ, Levens ED, Levy G, Ryan ME, Csokmay JM, DeCherney AH, et al. The use of 373 recombinant luteinizing hormone in patients undergoing assisted reproductive techniques with 374 advanced reproductive age: a systematic review and meta-analysis. Fertil Steril. 2012;97(5):1108-

375 14.e1.

376 33. Tehrani FR, Solaymani-Dodaran M, Hedayati M, Azizi F. Is polycystic ovary syndrome an 377 exception for reproductive aging? Hum Reprod. 2010;25(7):1775-81.

378 34. Carmina E, Campagna AM, Lobo RA. A 20-year follow-up of young women with polycystic 379 ovary syndrome. Obstet Gynecol. 2012;119(2 Pt 1):263-9.

380 35. Hwang YI, Cha SW, Song IO, Yang KM, Min EG, Kim HO. Fertility of patients with 381 polycystic ovary syndrome undergoing in vitro fertilization by age. Int J Gynaecol Obstet.

382 2016;135(1):91-5.

383 36. Meeting abstracts from the 2019 IFFS Shanghai world congress. Glob Reprod Health. 384 2019;4(1):e30. 
Tables

387 Table 1. Demographics and IVF/ICSI treatment characteristics of the women with tubal infertility 388 and PCOS

\begin{tabular}{|c|c|c|c|c|c|c|}
\hline & $\begin{array}{l}\text { PCOS } \\
(n=190)\end{array}$ & $\begin{array}{l}\text { Tubal infertility } \\
\qquad(\mathrm{n}=627)\end{array}$ & $\mathrm{t}$ & $\chi^{2}$ & $\mathrm{z}$ & $p$-value \\
\hline Age (y) & $36.93 \pm 2.19$ & $38.02 \pm 2.83$ & -4.895 & & & $<0.001^{*}$ \\
\hline Infertility duration (y) & $5.78 \pm 4.11$ & $5.15 \pm 4.33$ & & & 2.719 & $0.007 *$ \\
\hline BMI $\left(\mathrm{kg} / \mathrm{m}^{2}\right)$ & $24.43 \pm 3.40$ & $23.49 \pm 3.11$ & 3.600 & & & $<0.001 *$ \\
\hline LH/FSH & $1.01 \pm 1.10$ & $0.59 \pm 0.27$ & 8.217 & & & $<0.001 *$ \\
\hline Basal T (ng/dl) & $19.60 \pm 12.81$ & $18.63 \pm 11.94$ & 0.963 & & & 0.336 \\
\hline Total Gn (IU) & $2712.17 \pm 1247.43$ & $3320.32 \pm 1040.29$ & -6.7260 & & & $<0.001 *$ \\
\hline $\begin{array}{l}\text { rLH supplementation } \\
(\mathrm{Y} / \mathrm{N})(\%)\end{array}$ & $57.89 \%(110 / 80)$ & $36.84 \%(231 / 396)$ & & 26.576 & & $<0.001 *$ \\
\hline $\begin{array}{l}\text { Number of oocytes } \\
\text { retrieved }\end{array}$ & $12.87 \pm 6.67$ & $10.05 \pm 5.34$ & 5.985 & & 5.295 & $<0.001 *$ \\
\hline $\begin{array}{l}\text { High-quality embryo } \\
\text { rate (\%) }\end{array}$ & $\begin{array}{c}42.60 \% \\
(794 / 1866)\end{array}$ & $38.70 \%(1515 / 3916)$ & & 7.864 & & $0.006 *$ \\
\hline
\end{tabular}

389 Values are expressed as percentage (\%) or mean and standard deviation (SD). P-value <0.05 (asterisk) was 390 considered statistically significant. (IVF, in vitro fertilization; ICSI, intracytoplasmic sperm injection; PCOS,

391 Polycystic ovary syndrome; BMI, body mass index; LH, luteinizing hormone; FSH, follicle-stimulating hormone; T, 392 testosterone; Gn, gonadotropin; rLH, recombinant luteinizing hormone) 
395 Table 2. Demographics and IVF/ICSI treatment characteristics of the live-birth and the non-live396 birth groups of fresh cycles

\begin{tabular}{|c|c|c|c|c|c|c|}
\hline & $\begin{array}{l}\text { Live-birth group } \\
\qquad(\mathrm{n}=465)\end{array}$ & $\begin{array}{l}\text { Non-live-birth group } \\
\qquad(\mathrm{n}=352)\end{array}$ & $\mathrm{t}$ & $\chi^{2}$ & $\mathrm{z}$ & $p$-value \\
\hline $\operatorname{Age}(y)$ & $37.10 \pm 2.20$ & $38.64 \pm 3.11$ & 8.280 & & & $<0.001 *$ \\
\hline Infertility cause (PCOS/tubal) & $28.17 \%(131 / 334)$ & $16.76 \%(59 / 293)$ & & 14.616 & & $<0.001 *$ \\
\hline \multicolumn{7}{|l|}{$(\%)$} \\
\hline Infertility duration (y) & $5.14 \pm 4.10$ & $5.51 \pm 4.52$ & & & 0.882 & 0.378 \\
\hline \multirow[t]{2}{*}{ BMI $\left(\mathrm{kg} / \mathrm{m}^{2}\right)$} & $23.73 \pm 3.18$ & $23.68 \pm 3.24$ & - & & & 0.845 \\
\hline & & & 0.196 & & & \\
\hline \multirow[t]{2}{*}{ LH/FSH } & $0.73 \pm 0.74$ & $0.63 \pm 0.48$ & - & & & $0.038^{*}$ \\
\hline & & & 2.073 & & & \\
\hline \multirow[t]{2}{*}{ Basal T (ng/dl) } & $19.60 \pm 13.18$ & $17.86 \pm 10.58$ & - & & & $0.042 *$ \\
\hline & & & 2.036 & & & \\
\hline Total Gn (IU) & $3006.47 \pm 1123.15$ & $3406.67 \pm 1078.25$ & 5.131 & & & $<0.001 *$ \\
\hline rLH supplementation & $46.45 \%(216 / 249)$ & $35.51 \%(125 / 227)$ & & 9.861 & & $0.002 *$ \\
\hline \multicolumn{7}{|l|}{$(\mathrm{Y} / \mathrm{N})(\%)$} \\
\hline \multirow[t]{2}{*}{ Number of oocytes retrieved } & $12.22 \pm 5.96$ & $8.71 \pm 4.91$ & - & & & $<0.001 *$ \\
\hline & & & 8.981 & & & \\
\hline High-quality embryo rate (\%) & $43.40 \%(1699 / 3916)$ & $32.70 \%(610 / 1866)$ & & 60.275 & & $<0.001 *$ \\
\hline \multicolumn{7}{|c|}{$\begin{array}{l}\text { Values are expressed as percentage (\%) or mean and standard deviation (SD). P-value }<0.05 \text { (asterisk) was } \\
\text { considered statistically significant. (IVF, in vitro fertilization; ICSI, intracytoplasmic sperm injection; PCOS, } \\
\text { Polycystic ovary syndrome; BMI, body mass index; LH, luteinizing hormone; FSH, follicle-stimulating hormone; T, } \\
\text { testosterone ; Gn, gonadotropin; rLH, recombinant luteinizing hormone) }\end{array}$} \\
\hline
\end{tabular}


402 Table 3. Multivariable Cox regression analyses of CLBRs

\begin{tabular}{lllc}
\hline Independent covariates & HR & 95\% CI & $p$-value \\
\hline PCOS vs tubal & 0.96 & 0.77 to 1.20 & 0.727 \\
Infertility duration & 1.00 & 0.97 to 1.02 & 0.722
\end{tabular}

(every 1 additional year)

Age (above 37 vs below 37 years)

0.65

0.53 to 0.80

$<0.001 *$

Number of oocytes retrieved

1.08 to 1.59

$0.005^{*}$

(above 10 vs below 10)

High-quality embryo rate (above 0.3 vs

below 0.3 )

rLH supplementation vs no rLH

BMI (above 24 vs below 24)

LH on HCG day (above 1.2 vs below 1.2)

Basal T (above 12 vs below 12)

Gn (above 3000 IU vs below3000 IU)
1.25

0.99

1.02

1.16

0.85
1.03 to 1.53

0.82 to 1.20

0.918

0.83 to 1.24

0.883

403 P-value $<0.05$ (asterisk) was considered statistically significant. (CLBRs, cumulative live birth rates; PCOS,

Figure legends 


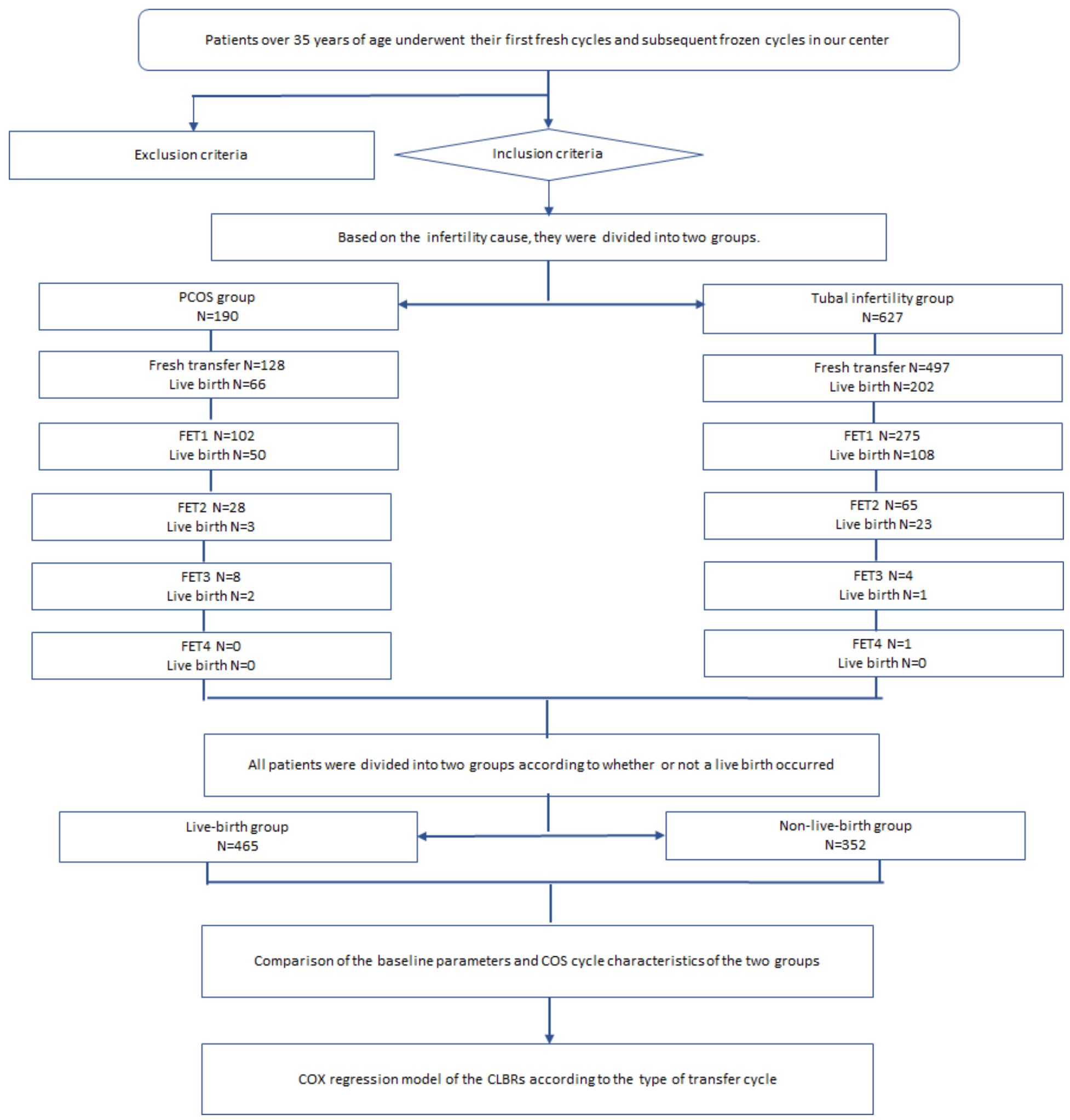

\section{Figure 1}

Trial flow chart. An overview of the patient selection and grouping. 